\title{
GloNet - das Netzwerk für Globales Lernen in der beruflichen Bildung
}

Bereits im Jahr 2014 hat das EPIZ Berlin das bundesweite Vernetzungstreffen GloNet ins Leben gerufen, um Organisationen mit dem Fokus auf Globales Lernen für die berufliche Bildung in Austausch miteinander zu bringen. Das Ziel der Treffen ist es, uns gegenseitig über unsere Arbeit zu informieren, voneinander zu lernen und gemeinsame Diskussionen über aktuelle Entwick- lungen im Bereich der beruflichen Bildung zu führen. Wir wollen dabei gemeinsame Herausforderungen ebenso ausloten wie die Kooperationsmöglichkeiten untereinander. Deutschlandweit sind heute viele Organisationen im Bereich des Globalen Lernens aktiv. Berufliche Bildung steht dabei nur bei sehr wenigen im Fokus - obwohl über 50\% aller Schülerinnen und Schü- 
ler in Deutschland eine Ausbildung beginnen. Sie sind somit eine besonders wichtige Zielgruppe für Globales Lernen und BNE. Denn sie können nicht nur für zivilgesellschaftliches Engagement und kritischen Konsum sensibilisiert werden, sondern auch für nachhaltiges Wirtschaften in ihrem Betrieb und ihrer Branche.

Für Organisationen, bei denen Globales Lernen mit Zielgruppen der Beruflichen Bildung einen Schwerpunkt ihrer Arbeit darstellt, ist die Vernetzung miteinander eine große Chance: Die Herausforderungen, denen sie gegenüber stehen, sind häufig andere als in der allgemeinbildenden Schule. Lehrkräfte wünschen sich Anknüpfungspunkte dicht am Ausbildungsberuf und eine hohe Fachspezifik. Auch die duale Ausbildung, bei der die Schülerinnen und Schüler meist nur ein oder zwei Tage pro Woche in der Schule sind, stellt außerschulische Träger mit ihren Angeboten vor besondere Herausforderungen in der Kooperation. Das Thema ,heterogene Zielgruppen" betrifft die berufliche Bildung in besonderem Maße, da sich in derselben Klasse unter Umständen sowohl ehemalige Schulverweigerer/-innen als auch Studienabbrecher/-innen befinden können. Sich z.B. mit den eigenen berufsbiographischen Erfahrungen im globalen Kontext auseinanderzusetzen und sich über Lösungsstrategien auszutauschen, wird von allen teilnehmenden Organisationen als befruchtend wahrgenommen: „Der Mehrwert unserer Treffen ist absolut gegeben“, berichtet eine GloNet-Teilnehmerin über ihre Erfahrungen mit dem Netzwerk. „Allein dadurch, dass wir Lehrkräfte aus den beruflichen Schulen über weitere Projekte zu Nachhaltigkeitsthemen passgenau informieren können, lässt schon Synergien entstehen“.

Die Treffen, die etwa alle zehn Monate stattfinden, werden meist im EPIZ-Berlin durchgeführt. Wenn Ihre Organisation auch Globales Lernen für die berufliche Bildung anbietet und Sie Interesse haben, an den Vernetzungstreffen teilzunehmen, melden Sie sich gerne bei uns unter Tel. 030/69264-19, Mail: epiz@epiz-berlin.de. Aktuell sind unter anderem folgende Organisationen, Personen und Projekte bei GloNet vertreten:

BUKO Pharma-Kampagne: Die BUKO Pharma-Kampagne setzt sich für das Menschenrecht auf Gesundheit ein, für eine global gerechte Arzneimittelversorgung und für eine bedürfnisorientierte Forschung, die auch die Krankheiten der Armen im Blick hat. Unterrichtsmaterialien, Unterrichtsbesuche und Theateraufführungen an weiterführenden Schulen und Berufsschulen sind Teil der Bildungsarbeit.

Ensa-Programm - Schulaustausch für eine global gerechtere Welt: Das ENSA-Programm fördert den Austausch zwi- schen Schulgruppen aus Deutschland und Afrika, Asien, Lateinamerika oder Südosteuropa. Schulpartnerschaften werden sowohl finanziell, als auch mit einer pädagogisch-inhaltlichen Vor- und Nachbereitung unterstützt. Das ENSA-Programm möchte den internationalen partnerschaftlichen Austausch von Berufsschulen mit dem Globalen Süden im besonderen Maße unterstützen.

EPIZ Berlin, Arbeitsbereich Berufliche Bildung: Das EPIZ Berlin bietet berufsspezifische Bildungsmaterialien, Schulkooperationen, Veranstaltungen für Berlin-Brandenburger Schüler/-innen und bundesweit kostenlose Fortbildungen für Referent/-innen, Lehramtsanwärter/-innen und Lehrkräfte an.

EXILE Kulturkoordination e.V., KaffeeGartenRuhr: Mit dem „KaffeeGartenRuhr“ haben Promotor/-innen von EXILE und dem Eine Welt Netz mit dem Röster Alex Kunkel einen außerschulischen Lernort geschaffen der Lernangebote für die berufliche Bildung insbesondere für die Fachbereiche Ernährungs- und Versorgungsmanagement, Agrarwirtschaft, Wirtschaft und Verwaltung anbietet.

Fachpromotorin für Globales Lernen und Nachhaltigkeit in Hessen: Als Eine Welt-Promotorin berät Lydia Koblofsky hessenweit zu inhaltlichen wie auch pädagogischen Fragen des Globalen Lernens. Ein wichtiger Schwerpunkt ist dabei die Zusammenarbeit mit Berufsschulen und Volkshochschulen (Projekttage, Pädagogische Tage, Bildungsurlaub).

ICJA, Projekt GLOBE - Globales Lernen an Berufsschulen: Ehrenamtliche Rückkehrer/-innen aus Freiwilligendienstprogrammen des ICJA bieten in Berufs- und Fachoberschulen bundesweit Workshops zu Globalisierungsthemen an. Ziel ist es, die Schüler/-innen für Globalisierungsprozesse und ihre eigene berufliche sowie persönliche Rolle darin zu sensibilisieren.

World University Service, Projekt Grenzenlos - Globales Lernen in der beruflichen Bildung: Bei Grenzenlos vermitteln Studierende aus Afrika, Asien und Lateinamerika Themen des Globalen Lernens an berufsbildenden Schulen in Baden Württemberg, Brandenburg, Hessen, Rheinland-Pfalz und im Saarland im Unterricht, in Projektwochen oder an Projekttagen.

Silvia Kröhn und Dr. Julia Boger, World University Service - Deutsches Komitee e.V. boger@wusgermany.de www.wusgermany.de/de/auslaenderstudium/grenzenlos doi.org/10.31244/zep.2018.02.11 